\title{
Intra-Seasonal Short-Time Up-Dosing with SQ-Standardised Subcutaneous Immunotherapy in Patients with Intermittent Allergic Rhinoconjunctivitis is Well Tolerated during Routine Application: A Non-Interventional, Observational Study
}

\author{
Rainer Reiber ${ }^{1}$, Hendrik Wolf ${ }^{2 *}$, Jörg Schnitker ${ }^{3}$ and Eike Wüstenberg ${ }^{2}$ \\ ${ }^{1}$ ENT-Physician, Schorndorf, Germany \\ ${ }^{2}$ Clinical Development, Medical \& Regulatory Affairs, ALK-Abelló Arzneimittel GmbH, Hamburg, Germany
}

${ }^{3}$ Institut für angewandte Statistik $\mathrm{GmbH}$, Bielefeld, Germany

\begin{abstract}
Background: For patients with pollen induced allergic rhinoconjunctivitis, a pre-seasonal start of subcutaneous specific immunotherapy is generally recommended in international guidelines. In a placebo-controlled trial intraseasonal up-dosing of subcutaneous immunotherapy to $10,000 \mathrm{SQ}-\mathrm{U}$ (Alutard $\mathrm{SQ}^{\circledR}$ ) was shown to be well tolerated and to induce significant immunologic effects. The aim of our study was to investigate the feasibility of intra-seasonal up-dosing during routine application.
\end{abstract}

Methods: In an open, non-interventional observational study, data on tolerability of subcutaneous immunotherapy (Alutard $\mathrm{SQ}^{\circledR}$ ) in patients with grass pollen induced allergic rhinoconjunctivitis were recorded by 110 physicians between May and November 2009 in Germany. Therapy was initiated within grass pollen season according to a 6-injection up-dosing schedule from 100 to 10,000 SQ-U with 1-3 days intervals, repeating this dose after 2 and 4 weeks during and up-dosing to the final maintenance dose of 100,000 SQ-U after the end of grass pollen season.

Results: Data for 250 patients could be evaluated, 198 patients had been up-dosed up to the peak grass pollen season and 52 after the peak. Adverse drug reactions were observed in $61.6 \%$ of patients with up-dosing up to the peak grass pollen season and in $48.1 \%$ with up-dosing after the peak with swelling at the injection site as most common reaction. The overall rate of systemic allergic reactions was low in both groups. Mild to moderate respiratory symptoms were more frequently reported in patients up-dosed up to the peak grass pollen season. Overall tolerability was assessed as "very good" or "good" by $>90 \%$ of patients and physicians.

Conclusions: Intra-seasonal short-time up-dosing with Alutard SQ ${ }^{\circledR}$ grasses and rye by 6 injections from 100 to 10,000 SQ-U with 1-3 days interval was observed to be well-tolerated during routine application and thus confirms data obtained from the placebo-controlled clinical trial.

Keywords: Subcutaneous immunotherapy; Intra-seasonal updosing; Short-time up-dosing; Grass pollen allergy; Allergic rhinitis; Tolerability

Abbreviations: ADR: Adverse Drug Reaction; GPS: Grass Pollen Season; SCIT: Subcutaneous Specific Immunotherapy; SIT: Specific Allergen Immunotherapy; SQ: Standardized Quality

\section{Introduction}

Specific allergen immunotherapy (SIT) is regarded as the only treatment that is able to treat the underlying cause of the allergic disease [1]. Long-term efficacy and a disease modifying effect after a three year all year round course of treatment has been proven and approved by the European authorities for a grass allergen immunotherapy tablet with Phleum pratense allergens as active ingredient [2,3]. The asthma preventative and long term effect of subcutaneous specific immunotherapy (SCIT) has been shown for a SCIT-product using intact allergens from birch or grasses [4]. International and national German guidelines recommend that up-dosing of SIT against seasonal allergens should be started outside the pollen season [5-7]

Patients with seasonal allergies visit the allergist's office usually during the pollen season when they have symptoms. Following these guidelines the physician can offer only symptomatic treatment and request patients to return for a causal treatment by SIT after the pollen season. Patients are likely to be less motivated for SIT once symptoms have declined after the season. Thus, many patients do not receive SIT.

For SIT with the grass immunotherapy tablet data from a controlled clinical trial have been published demonstrating that an intra-seasonal start of treatment within the grass pollen season is well tolerated and induces significant immunologic effects [8].

By a recent placebo-controlled study with SCIT, an intra-seasonal up-dosing schedule with 6-injections and 1-3 days intervals for an unmodified alum-adsorbed grasses and secale pollen preparation (Alutard SQ ${ }^{\circledR}$ ) has been established [9]. Intra-seasonal up-dosing by this schedule was shown to be well tolerated and to induce a significantly higher level of IgE-blocking components (IgE blocking factor) in serum.

*Corresponding author: Hendrik Wolf, ALK-Abelló Arzneimittel GmbH, Medical \& Regulatory Affairs, Griegstrasse 75 Haus 25, Hamburg, D-22763, Germany, Tel: +49 40 703845-342; Fax: +49 40 70384555-30; E-mail: hendrik.wolf@alk.net

Received May 03, 2013; Accepted June 15, 2013; Published June 20, 2013

Citation: Reiber R, Wolf H, Schnitker J, Wüstenberg E (2013) Intra-Seasonal Short-Time Up-Dosing with SQ-Standardised Subcutaneous Immunotherapy in Patients with Intermittent Allergic Rhinoconjunctivitis is Well Tolerated during Routine Application: A Non-Interventional, Observational Study. J Allergy Ther 4: 137. doi:10.4172/2155-6121.1000137

Copyright: ( 2013 Reiber R, et al. This is an open-access article distributed unde the terms of the Creative Commons Attribution License, which permits unrestricted use, distribution, and reproduction in any medium, provided the original author and source are credited. 
Citation: Reiber R, Wolf H, Schnitker J, Wüstenberg E (2013) Intra-Seasonal Short-Time Up-Dosing with SQ-Standardised Subcutaneous Immunotherapy in Patients with Intermittent Allergic Rhinoconjunctivitis is Well Tolerated during Routine Application: A Non-Interventional, Observational Study. J Allergy Ther 4: 137. doi:10.4172/2155-6121.1000137

Based on these data this intra-seasonal up-dosing schedule with Alutard $\mathrm{SQ}{ }^{\circledR}$ grasses has been approved for routine use by the German regulatory authority, the Paul-Ehrlich-Institute [10].

Systematically recorded data on the application and tolerability of the intra-seasonal up-dosing schedule in real life are needed to evaluate the benefit and risks in daily use. Objective of our non-interventional observational study was, therefore, to investigate the feasibility and experiences made during the routine use of the new intra-seasonal short-time up-dosing schedule under real life conditions.

\section{Methods}

\section{Study design}

This study was performed as a non-interventional, non-randomised, open-label, observational study to investigate the feasibility of an intraseasonal short-time up-dosing schedule up to 10,000 SQ-U for Alutard $\mathrm{SQ}^{\circledR}$ grasses and secale (ALK, Hørsholm, Denmark) within the grass pollen season (GPS) that is recommended for routine use [10]. All research procedures were realized according to ethical guidelines.

Alutard $\mathrm{SQ}^{\circledR}$ is a range of purified, qualitatively and quantitatively standardized allergen immunotherapy products in a sustained-release formulation for subcutaneous injection, for the treatment of allergic rhinoconjunctivitis and mild to moderate allergic asthma. Alutard $\mathrm{SQ}^{\circledR}$ contains a suspension of equal quantities of allergens from 6 grasses and rye (Dactylis glomerata, Lolium perenne, Avena elatior, Phleum pratense, Poa pratensis, Festuca pratensis and Secale cereale) adsorbed to aluminium hydroxide. The allergen extracts are expressed in standardized quality (SQ) units. The dose of 10,000 SQ-U was scheduled to be maintained during grass pollen season (GPS) and the dose to be increased to the recommended maintenance dose of 100,000 SQ-U as constant dose for maintenance treatment after the end of GPS.

The centres participating in this study were distributed evenly across Germany. For this purpose allergists applying specific immunotherapy were registered in regional lists and asked for participation in the study in a randomly arranged new order. In case of participation, the centres were asked to record data of 3 patients in a consecutive order dependent on the patient's willingness to participate in the study. Together, the random selection of centres and the consecutive choice of study patients represent a suitable measure to avoid a selection bias in the study population. Physicians were asked to document all patients who were potentially eligible for the study in a patient log, thus, the selection process remained fully transparent.

All patients with type I-allergy caused by grass pollen allergens and indication for specific immunotherapy were eligible to be documented for the study after the decision to apply SCIT with Alutard SQ ${ }^{\circledR}$ grasses and secale within the GPS had been taken independently from the study. The study was conducted in 110 allergological centres in Germany in the period between May and November 2009 and included 250 patients suffering predominantly from allergic rhinitis and conjunctivitis with or without asthma.

The physicians were instructed to document the injections applied for up-dosing during GPS following the recommended schedule and the first two maintenance injections with 100,000 SQ-U, if applied during the observation period, and to record any adverse reactions. The recommended dosage schedule is displayed in Figure 1. Patients were scheduled to be up-dosed by 6 injections with 1 to 3 day intervals up to a dose level of 10,000 SQ-U. During the course of GPS further injections with 10,000 SQ-U should be applied with a 2 weeks and then

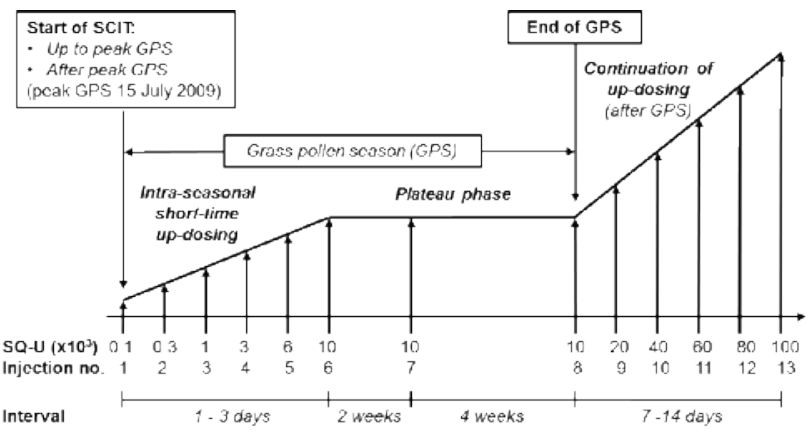

Figure 1: Dosage scheme for updosing with specific immunotherapy (SCIT) Patients started SCIT intra-seasonally and were stratified according to a start up to the peak of the grass pollen season 'up to peak GPS' (with increasing pollen exposure) or 'after peak GPS' (with declining pollen exposure). As peak season 15 July was defined for GPS 2009 in Germany. Patients were scheduled to be up-dosed to a dose of 10,000 SQ-U with 1-3 day intervals. Treatment was continued with subsequent injections of 10.000 SQ-U (plateau phase) until the end of GPS. After the end of GPS up-dosing was continued to 100.000 SQ-U using 7-14 day intervals as maintenance dose for the intended 3 years course of SCIT.

\begin{tabular}{|c|c|c|c|}
\hline Time of up-dosing & Up to peak GPS & After peak GPS & Total \\
\hline Total number of patients, $\mathrm{N}$ & 198 & 52 & 250 \\
\hline Median age (range), years & $26.5(6-73)$ & $29.5(5-50)$ & $27.0(5-73)$ \\
\hline \multicolumn{4}{|l|}{ Gender, N (\%) } \\
\hline males & $101(51.0 \%)$ & $23(44.2 \%)$ & $124(49.6 \%)$ \\
\hline females & $97(49.0 \%)$ & $29(55.8 \%)$ & $126(50.4 \%)$ \\
\hline $\begin{array}{l}\text { Duration since diagnosis of } \\
\text { grass pollen allergy, mean } \pm \\
\text { SD, years }\end{array}$ & $3.2 \pm 5.4$ & $5.5 \pm 9.7$ & $3.7 \pm 6.6$ \\
\hline \multicolumn{4}{|l|}{$\begin{array}{l}\text { Patients with moderate to } \\
\text { severe symptoms, N (\%) }\end{array}$} \\
\hline Nasal symptoms & $183(92.4 \%)$ & $49(94.3 \%)$ & $232(92.8 \%)$ \\
\hline Eye symptoms & $163(82.3 \%)$ & $42(80.8 \%)$ & $205(82.0 \%)$ \\
\hline $\begin{array}{l}\text { Patients withknown } \\
\text { concomitant allergies, N (\%) }\end{array}$ & $116(60.1 \%)$ & $38(74.5 \%)$ & $154(63.1 \%)$ \\
\hline tree pollen & $82(41.4 \%)$ & $23(44.2 \%)$ & $105(42.0 \%)$ \\
\hline house dust mites & $43(21.7 \%)$ & $17(32.7 \%)$ & $60(24.0 \%)$ \\
\hline animal epithelia & $38(19.2 \%)$ & $11(21.2 \%)$ & $49(19.6 \%)$ \\
\hline Patients with asthma, N (\%) & $43(21.7 \%)$ & $10(19.2 \%)$ & $53(21.2 \%)$ \\
\hline $\begin{array}{l}\text { Patients first treated with SIT, } \\
\mathrm{N}(\%)\end{array}$ & $176(88.9 \%)$ & $43(82.7 \%)$ & $219(87.6 \%)$ \\
\hline $\begin{array}{l}\text { Patients who used } \\
\text { symptomatic drugs in previous } \\
\text { GPS }\end{array}$ & $168(84.8 \%)$ & $44(84.6 \%)$ & $212(84.8 \%)$ \\
\hline
\end{tabular}

Table 1: Patient characteristics stratified for patients who started treatment up to the peak of the grass pollen season ('up to peak GPS') or after the end of the grass pollen season ('after peak GPS')

4 weeks interval (plateau phase). After the end of GPS patients were scheduled to be up-dosed to the final maintenance dose of 100,000 SQ-U by 5 injections with 7-14 days intervals.

Additionally, the physicians were asked to record the following items in the case report forms: Allergy history, applied diagnostics, symptoms at nose, eyes, bronchi and skin (none/mild/moderate/severe), and medication use in the GPS before start of SCIT and medication applied concomitantly with SCIT. The injections were recorded with date, time, volume and vial number and it was documented whether an anti-allergic premedication had been administered. Adverse drug reactions (ADRs) were recorded as local reactions at the injection site with size of injection site swelling (no reaction/swelling: $\leq 5 \mathrm{~cm} ;>5 \mathrm{~cm}$ and $<8 \mathrm{~cm} ; \geq 8 \mathrm{~cm}$ ) or as systemic reactions. Systemic reactions were 
Citation: Reiber R, Wolf H, Schnitker J, Wüstenberg E (2013) Intra-Seasonal Short-Time Up-Dosing with SQ-Standardised Subcutaneous Immunotherapy in Patients with Intermittent Allergic Rhinoconjunctivitis is Well Tolerated during Routine Application: A Non-Interventional, Observational Study. J Allergy Ther 4: 137. doi:10.4172/2155-6121.1000137

Page 3 of 6

classified according to time after injection, intensity (mild/moderate/ severe), causality (not related/unlikely/possible/probable/definite/ unknown) and reversibility (yes/no). ADRs were coded using the Medical Dictionary for Regulatory Activities (MedDRA).

\section{Results}

\section{Patients}

Data for 250 patients with rhinoconjunctivitis and a median age of 27.0 years could be evaluated who started treatment from May 2009 within GPS. Patients were equally distributed with respect to gender. The majority of the patients had moderate to severe nose and eye symptoms and had used symptomatic medication in the previous GPS; $21.2 \%$ patients had asthma (Table 1 ).

\begin{tabular}{|c|c|}
\hline Start of short-time up-do & \\
\hline to $10,000 S Q-U$ & $\mathrm{~N}=250$ \\
\hline - $\quad$ Up to peak GPS & $\mathrm{N}=198(79.2 \%)$ \\
\hline - $\quad$ After peak GPS & $\mathrm{N}=52(20.8 \%)$ \\
\hline
\end{tabular}

\begin{tabular}{|cl|}
\hline Discontinued & $\mathrm{N}=27(10.8 \%)$ \\
- Adverse events & $\mathrm{N}=8(3.2 \%)$ \\
- Not returned & $\mathrm{N}=9(3.6 \%)$ \\
- $\quad$ Moved & $\mathrm{N}=1(0.4 \%)$ \\
- Documentation discontinued & $\mathrm{N}=9(3.6 \%)$ \\
\hline & \\
\end{tabular}

Continued after short-time up-dosing $\quad \mathrm{N}=223(89.2 \%)$

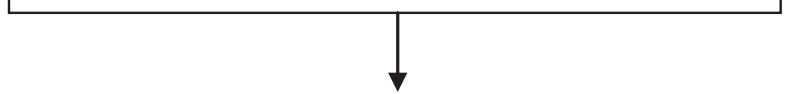

\begin{tabular}{|c|c|}
\hline Discontinued & $\mathrm{N}=22(8.8 \%)$ \\
\hline - Adverse events & $\mathrm{N}=1(0.4 \%)$ \\
\hline - Not returned & $\mathrm{N}=6(2.4 \%)$ \\
\hline - $\quad$ Moved & $\mathrm{N}=1(0.4 \%)$ \\
\hline - Documentation discontinued & $\mathrm{N}=14(5.6 \%)$ \\
\hline Up-dosing to $100,000 \mathrm{SQ}-\mathrm{U}$ & $\mathrm{N}=201(80.4 \%)$ \\
\hline
\end{tabular}

Figure 2: Flow of patients through the study.
For evaluation patients were stratified according to their start of treatment related to the peak of GPS 2009 in Germany. In 198 patients up-dosing had been started early after initiation of the study 'up to peak GPS' (defined as 15 July 2009) and in 52 patients later ('after peak GPS') when grass pollen exposure was already declining. The flow of patients through the study is displayed in Figure 2. For the total number of 250 patients who initially started treatment with short-time up-dosing, injections were recorded up to the dose level of 10,000 SQ-U and subsequent injections in $223(89.2 \%)$ patients. Further injections with 10,000 SQ-U during GPS (plateau phase) were recorded in $152(68.2 \%)$ patients and the up-dosing to the maintenance dose of 100,000 SQ-U in $201(80.4 \%)$ patients. Maintenance injections with 100,000 SQ-U were documented for $70(28.0 \%)$ patients. In patients who started treatment 'after peak GPS' less injections were documented than in patients who started treatment 'up to peak GPS' because the study was terminated at the end of November 2009 for all patients included.

\section{Application of the recommended short-time up-dosing schedule}

The recommended dosage schedule for short-time up-dosing to $10,000 \mathrm{SQ}-\mathrm{U}$ was followed in most of the patients. Six injections according to the recommended schedule were administered in 200 (80.0\%) of the 250 total patients, in $38(15.2 \%)$ more than 6 injections had been applied and less than 6 injections in $12(4.8 \%)$ patients. In the group who started therapy 'up to peak GPS', 6 injections according to the recommended schedule were applied in 159 (80.3\%) patients and in $33(63.5 \%)$ patients in the group with treatment start 'after peak GPS. Additional dosage steps have been applied in $11.2 \%$ of patients, dosage steps repeated in $4.4 \%$ and dosage steps have been skipped in $0.8 \%$ of patients $(4.0 \%$ discontinued treatment, in $2.8 \%$ of patients documentation was stopped).

For the short-time up-dosing phase up to 10,000 SQ-U 1,313 injection intervals were recorded, 1,038 'up to peak GPS' and 275 'after peak GPS'. Injection intervals of 1 to 3 days as recommended were recorded in $78.5 \%$ of total intervals performed, and intervals of 4 to 7 days in $14.5 \%$ and $>7$ days in $7.0 \%$ of total intervals (median interval 3.0 days). Injection intervals of 1 to 3 days were recorded in $79 \%$ of total intervals for patients who started treatment 'up to peak GPS' and in $76.4 \%$ of total intervals for patients who started treatment 'after peak GPS.

The dose level of 10,000 SQ-U has been reached in 221 (88.4\%) patients in total and in $182(91.9 \%)$ patients with start of treatment 'up to peak GPS' and in 39 (75.0\%) with start of treatment 'after peak GPS'. In $152(68.2 \%)$ patients out of 223 patients who continued treatment after short-time up-dosing to $10,000 \mathrm{SQ}-\mathrm{U}$ further injections on a constant dose level of 10,000 SQ-U were applied as recommended; $71.0 \%$ patients received $2-3$ injections, $12.5 \% 1$ injection and $16.4 \%$ more than 4 injections. The start of the up-dosing to the final maintenance dose of 100,000 SQ-U was documented in 201 (80.4\%) patients.

\section{Tolerability data}

The rate of patients and the rate of injections with ADRs for the entire observation period and the treatment phases with different dose levels are shown in Table 2. Treatment has been discontinued during or immediately after short-time up-dosing in 8 patients (3.2\%) who started up-dosing 'up to peak GPS'. Injection site swellings for the entire observation period as well as for short-time up-dosing to 10,000 SQ-U and systemic reactions for the entire observation period are displayed in Table 3. Systemic reactions were reported in more patients to be 
Citation: Reiber R, Wolf H, Schnitker J, Wüstenberg E (2013) Intra-Seasonal Short-Time Up-Dosing with SQ-Standardised Subcutaneous Immunotherapy in Patients with Intermittent Allergic Rhinoconjunctivitis is Well Tolerated during Routine Application: A Non-Interventional, Observational Study. J Allergy Ther 4: 137. doi:10.4172/2155-6121.1000137

Page 4 of 6

\begin{tabular}{|c|c|c|c|c|c|c|}
\hline \multirow[t]{2}{*}{ Start of up-dosing } & \multicolumn{2}{|c|}{ Up to peak GPS } & \multicolumn{2}{|c|}{ After peak GPS } & \multicolumn{2}{|c|}{ Total } \\
\hline & $\begin{array}{c}\text { Patients } \\
\text { ADR/Total, N (\%), } E^{*}\end{array}$ & $\begin{array}{c}\text { Injections } \\
\text { ADR/Total, N (\%) }\end{array}$ & $\begin{array}{c}\text { Patients } \\
\text { ADR/Total, N (\%), } E^{*}\end{array}$ & $\begin{array}{c}\text { Injections } \\
\text { ADR/Total, N (\%) }\end{array}$ & $\begin{array}{c}\text { Patients } \\
\text { ADR/Total, N (\%), } E^{*}\end{array}$ & $\begin{array}{c}\text { Injections } \\
\text { ADR/Total, N (\%) }\end{array}$ \\
\hline Entiretreatment & 122/198 (61.6), 897 & $764 / 2396(31.9)$ & 25/52 (48.1), 208 & $163 / 526(31.0)$ & $147 / 250$ (58.8), 1105 & $927 / 2922(31.7)$ \\
\hline Up-dosing to $10,000 \mathrm{SQ}-U$ & 105/198 (53.0), 439 & $350 / 1236(28.3)$ & 24/52 (46.2), 124 & $97 / 324(29.7)$ & 129/250 (51.6), 563 & $447 / 1560(28.7)$ \\
\hline Plateau with $10,000 \mathrm{SQ}-\mathrm{U}$ & $45 / 134$ (33.6), 123 & $97 / 342(28.4)$ & 4/18 (22.2), 8 & $8 / 37(43.2)$ & 49/152 (32.2), 131 & $105 / 379(27.7)$ \\
\hline Up-dosing to $100,000 \mathrm{SQ}-\mathrm{U}$ & 81/164(49.4), 293 & $277 / 723(38.3)$ & 16/37 (43.2), 65 & $55 / 152(36.2)$ & 97/201 (48.3), 349 & $332 / 875(37.9)$ \\
\hline $\begin{array}{l}\text { Maintenance with } 100,000 \\
\text { SQ-U }\end{array}$ & 28/62 (45.2), 42 & $40 / 95(42.1)$ & 2/8 (25.0), 3 & $3 / 10(30.0)$ & $30 / 70$ (42.9), 45 & $43 / 105(40.6)$ \\
\hline
\end{tabular}

*E: Number of events

Table 2: Frequency of Adverse Drug Reactions (ADRs) in patients and injections in the different phases of therapyfor patients who started treatment up to the peak of the grass pollen season ('up to peak GPS') or after the end of the grass pollen season ('after peak GPS') and total patients.

\begin{tabular}{|l|c|c|c|}
\hline & \multicolumn{2}{|c|}{ Start of updosing } & \\
\hline & Up to peak GPS & After peak GPS & Total \\
\hline & $\mathbf{N}(\%) \mathbf{E}^{*}$ & $\mathbf{N}(\%) \mathbf{E}^{*}$ & $\mathbf{N}(\%) \mathbf{E}^{*}$ \\
\hline Total number of patients & 198 & 52 & 250 \\
\hline $\begin{array}{l}\text { Injection site swelling } \\
\text { (entire observation period) }\end{array}$ & $112(56.6 \%) 734$ & $25(48.1 \%) 162$ & $137(54.8 \%) 896$ \\
\hline $\begin{array}{l}\text { Injection site swelling } \\
\text { (short-time up-dosing to } \\
\text { 10,000 SQ-U) }\end{array}$ & $98(49.5 \%) 326$ & $24(46.2 \%) 96$ & $122(48.8 \%) 422$ \\
\hline $\begin{array}{l}\text { Other ADR (entire } \\
\text { observation period) }\end{array}$ & $47(23.7 \%) 163$ & $6(11.5 \%) 46$ & $53(21.2 \%) 209$ \\
\hline$\leq 30$ min after injection & $11(5.6 \%) 46$ & $1(1.9 \%) 3$ & $12(4.8 \%) 49$ \\
\hline$>30$ min after injection & $31(15.7 \%) 98$ & $3(5.8 \%) 10$ & $34(13.6 \%) 108$ \\
\hline Missing values & $5(2.5 \%) 19$ & $2(3.8 \%) 33$ & $7(2.8 \%) 52$ \\
\hline Intensity & & & \\
\hline mild & $24(12.1 \%) 94$ & $1(1.9 \%) 34$ & $25(10.0 \%) 128$ \\
\hline moderate & $16(8.1 \%) 48$ & $1(1.9 \%) 5$ & $17(6.8 \%) 53$ \\
\hline severe & $5(2.5 \%) 9$ & $4(7.7 \%) 7$ & $9(3.6 \%) 16$ \\
\hline \multicolumn{1}{|c|}{ missing values } & $2(1.0 \%) 19$ & - & $2(0.8 \%) 12$ \\
\hline Treated by medication & $30(15.2 \%) 100$ & $5(9.6 \%) 35$ & $35(14.0 \%) 120$ \\
\hline Serious & $4(2.0 \%) 10$ & $1(1.9 \%) 4$ & $5(2.0 \%) 14$ \\
\hline Treatment terminated & $7(3.5 \%) 12$ & - & $7(2.8 \%) 16$ \\
\hline & & & \\
\hline & & & \\
\hline
\end{tabular}

\section{${ }^{*} \mathrm{E}$ : Number of events}

Table 3: Overview of Adverse Drug Reactions (ADRs) in patients who started treatment up to the peak of the grass pollen season ('up to peak GPS') or after the end of the grass pollen season ('after peak GPS') and in total patients.

delayed ( $\geq 30$ minutes after injection) than occurring immediately (within 30 minutes) after the injection. The majority of the reactions were of mild to moderate intensity, in $3.6 \%$ patients reactions were assessed as severe.

For the short-time up-dosing with 6 injections total ADRs were reported in 105 out of 198 (53.0\%) patients who started treatment 'up to peak GPS' and in 350 out of 1236 (28.3\%) injections. In patients who started treatment 'after peak GPS', ADRs were reported in 24 out of 52 (46.2\%) patients and in 97 out of 327 (29.7\%) injections.

The injection site swellings had a size $\leq 5 \mathrm{~cm}$ in $34.8 \%$ patients, in $14.8 \%$ a size $>5 \mathrm{~cm}$ and $<8 \mathrm{~cm}$ and in $5.2 \% \geq 8 \mathrm{~cm}$. Frequencies of injection site swellings of size $\leq 5 \mathrm{~cm}$ were observed in $36.9 \%$ patients who started treatment 'up to peak GPS' ( $>5 \mathrm{~cm}$ and $<8 \mathrm{~cm}: 5.7 \%, \geq 8 \mathrm{~cm}$ : $4.0 \%$ patients) and in $26.9 \%$ patients who started treatment 'after peak GPS' ( $>5 \mathrm{~cm}$ and $<8 \mathrm{~cm}: 11.5 \%, \geq 8 \mathrm{~cm}: 9.6 \%$ patients). The differences between the two patient groups were not significant when tested by means of the $\chi$-square test. The frequencies of injections with different size of swellings were not different for the two groups (Table 4).

The pattern of the ADRs reported during up-dosing to 10,000 SQ-U

\begin{tabular}{|l|c|c|}
\hline & \multicolumn{2}{|c|}{ SCIT injections, N (\%) } \\
\hline Size of swellings, cm & $\begin{array}{c}\text { Up to peak GPS } \\
\text { N=2396 }\end{array}$ & $\begin{array}{c}\text { After peak GPS } \\
\mathbf{N = 5 2 6}\end{array}$ \\
\hline No reaction & $1662(69.4)$ & $364(69.2)$ \\
\hline$>0 \mathrm{~cm}, \leq 5 \mathrm{~cm}$ & $623(26.0)$ & $137(26.0)$ \\
\hline$>5 \mathrm{~cm},<8 \mathrm{~cm}$ & $97(4.0)$ & $16(3.0)$ \\
\hline$\geq 8 \mathrm{~cm}$ & $14(0.6)$ & $9(1.7)$ \\
\hline
\end{tabular}

Table 4: Swellings at injection sites related to the number of injections with start of subcutaneous immunotherapy (SCIT) up to the peak of the grass pollen season ('up to peak GPS') and with start of SCIT after the peak of the grass pollen season ('after peak GPS')

is displayed in Figure 3. Most frequent reactions were injection site swellings as local reactions at the injection site. Respiratory symptoms as cough, dyspnoea, asthma and allergic rhinitis were reported as systemic reactions with low frequency, but were observed only in the group of patients who started treatment 'up to peak GPS'. Pruritus was observed in both groups as systemic reaction in $\geq 1 \%$ patients.

Serious reactions were reported in 5 cases. An anaphylactic reaction with generalised urticaria, dizziness and itching in a 15-yearold female patient during short-time intra-seasonal up-dosing and start of SCIT 'after peak GPS', 45 minutes after injection of 10,000 SQ-U and cough, dyspnoea and hives in a 17-year-old female 2 hours after injection of 10,000 SQ-U (intra-seasonal up-dosing, start 'up to peak GPS') were considered as medically important. Dyspnoea and urticaria were reported in a 17-year-old male patient 45 minutes after injection of 20,000 SQ-U (continuation of up-dosing after GPS), and dyspnoea in a 22 -year-old male patient 50 minutes after injection of 10,000 SQ-U (intra-seasonal up-dosing, start 'up to peak GPS'). Patients recovered after treatment with corticosteroids and antihistamines applied intravenously. Patients were hospitalised overnight for observation and continued treatment.

Laryngeal oedema, urticaria, flushing and cough were reported in a 29 -year-old female patient, 20 minutes after injection of 10,000 SQ-U (intra-seasonal up-dosing, start 'up to peak GPS'); the patient recovered after treatment with corticosteroids and antihistamines applied intravenously. She was hospitalised overnight for observation and discontinued treatment.

\section{Discussion}

This study was initiated to evaluate tolerability of SCIT applied within the grass pollen season under real-life conditions. The prospective, open-label observational design was appropriate to record data on the routine use of SCIT in a real-life setting.

Positive results from a placebo-controlled clinical trial on pharmacodynamic efficacy, safety and tolerability using the same 
Citation: Reiber R, Wolf H, Schnitker J, Wüstenberg E (2013) Intra-Seasonal Short-Time Up-Dosing with SQ-Standardised Subcutaneous Immunotherapy in Patients with Intermittent Allergic Rhinoconjunctivitis is Well Tolerated during Routine Application: A Non-Interventional, Observational Study. J Allergy Ther 4: 137. doi:10.4172/2155-6121.1000137

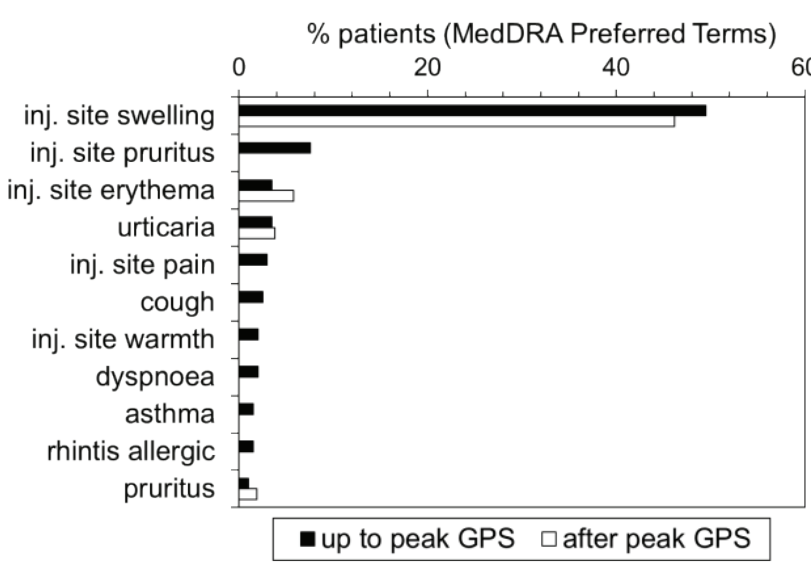

Figure 3: Most frequent adverse drug reactions (ADRs) during intraseasonal up-dosing ( $\geq 1 \%$ of patients, total patients) for patients who started subcutaneous immunotherapy (SCIT) up to the peak of the grass pollen season ('up to peak GPS') or after the peak of the grass pollen season ('after peak GPS'). ADRs were coded according to the Medical Dictionary for Regulatory Activities (MedDRA)

dosage regimen for intra-seasonal up-dosing performed in 2008 [9] were the basis for the German authority (Paul-Ehrlich-Institut) to grant a marketing authorisation for the intra-seasonal start of therapy with Alutard $\mathrm{SQ}^{\circledR}$ grasses as an extension to the existing marketing authorization of the product in Germany [10]. Data from the clinical trial showed an increased rate of local reactions compared with placebo, mainly as swelling at the injection site, but only small incidences of systemic allergic reactions with the active treatment [9].

For the current study data of 250 patients with allergy to grass pollen who started treatment with SCIT within the grass pollen season could be evaluated. The data were stratified into two groups of patients who started treatment at different times related to the peak of the grass pollen season; 198 patients started up-dosing in the period of grass pollen season until 15 July 2009 as average peak of the grass pollen season in Germany, and 52 patients thereafter. This stratification was performed to assure that all patients in the group with start of treatment 'up to the peak GPS' completed their short-time up-dosing phase within the grass pollen season 2009. These patients were higher exposed to grass pollen than the patients who started up-dosing 'after peak GPS' with already declining pollen exposure.

The recommended short-time up-dosing schedule of 6 injections to 10,000 SQ-U with 1-3 day intervals was applied in the majority of patients. Short-time up-dosing within the GPS was generally well tolerated. The rate of patients who discontinued treatment during short-time up-dosing was low (3.2\%). Most frequent ADRs were local swellings at the injection site (54.8\% of patients) with a size of $<5 \mathrm{~cm}$ ( $34.8 \%$ of patients), larger swellings up to $8 \mathrm{~cm}$ ( $14.8 \%$ of patients) and $>8 \mathrm{~cm}$ ( $5.2 \%$ of patients) were observed in less patients. Comparing the number of injections with swellings at the injection site for the group of patients who started SCIT 'up to peak GPS' and patients who started SCIT 'after peak GPS' no differences were detected.

An increased risk for local reactions compared to placebo was also observed in the controlled clinical trial conducted previously [9]. The differences in the percentages of patients with ADRs between the two strata in our study may primarily be induced by the longer observation period in the group who started treatment earlier ('up to peak GPS').
Other ADRs than injection site swellings were recorded in $21.2 \%$ of patients during the entire observation period. Severity was assessed as mild to moderate in the majority of patients. ADRs were assessed as serious in 5 patients (2.0\%) in total and in 4 patients during shorttime up-dosing with no difference in the rate of patients who started SCIT 'up to peak GPS' or 'after peak GPS'. The overall rate of patients who developed systemic reactions during intra-seasonal short-time up-dosing to 10,000 SQ-U was low. Most frequently the symptoms of allergic asthma and allergic rhinitis were observed in the group of patients who started SCIT 'up to peak GPS'. Since allergic asthma and allergic rhinitis are symptoms that patients are expected to have during GPS, the declining pollen exposure in the group who started SCIT 'after peak GPS' might also explain the lower number of asthmatic and rhinitis symptoms recorded as ADRs in this group.

The pattern of ADRs observed in our study was in line with previous randomised controlled clinical trials using non-modified alum-adsorbed grass pollen allergens $[11,12]$.

The duration of the disease since the first occurrence of allergic complaints (mean 3.7 years) appears to be shorter when compared with respective data from another observational study with SCIT in which treatment was started after the pollen season [13]. This observation indicates that more patients may potentially be treated earlier in the natural course of their disease with specific immunotherapy when patients are offered to start SIT immediately while visiting the doctor's office with acute symptoms during GPS.

Limitations of our study are those of a prospective, open-label, uncontrolled observational study. In order to minimize potential selection bias, sites evenly distributed all over Germany were involved that were selected from randomly ordered regional lists of allergists; these physicians were requested to include patients willing to participate in consecutive order.

In conclusion intra-seasonal up-dosing in this study was well tolerated during routine application in the allergist's practice and our results are well comparable with data for tolerability obtained during up-dosing outside the pollen season. Our data for the routine use of the product support the results of the previously conducted randomised controlled clinical trial. Therefore, this study confirmed that intraseasonal up-dosing is feasible for routine treatment, Intra-seasonal up-dosing may, therefore, be a good alternative to treatment initiation outside the pollen season possibly leading to an earlier treatment initiation of specific immunotherapy.

\section{Acknowledgments}

The authors would like to thank all physicians who provided data on treatment of their patients for the study.

\section{References}

1. European Medicines Agency (2008) Guideline on the clinical development of products for specific immunotherapy for the treatment of allergic diseases.

2. Grazax (2010) Summary of product characteristics. Wedel, Germany: ALK.

3. Durham SR, Emminger W, Kapp A, de Monchy JG, Rak S, Scadding GK, et al. (2012) SQ-standardized sublingual grass immunotherapy: confirmation of disease modification 2 years after 3 years of treatment in a randomized trial. J Allergy Clin Immunol 129: 717-725.

4. Jacobsen L, Niggemann B, Dreborg S, Ferdousi HA, Halken S, et al. (2007) Specific immunotherapy has long-term preventive effect of seasonal and perennial asthma: 10-year follow-up on the PAT study. Allergy 62: 943-948.

5. Bousquet J, Van Cauwenberge P, Khaltaev N; Aria Workshop Group; World Health Organization (2001) Allergic rhinitis and its impact on asthma. J Allergy Clin Immunol 108: S147-S334. 
Citation: Reiber R, Wolf H, Schnitker J, Wüstenberg E (2013) Intra-Seasonal Short-Time Up-Dosing with SQ-Standardised Subcutaneous Immunotherapy in Patients with Intermittent Allergic Rhinoconjunctivitis is Well Tolerated during Routine Application: A Non-Interventional, Observational Study. J Allergy Ther 4: 137. doi:10.4172/2155-6121.1000137

6. Bousquet J, Lockey R, Malling HJ (1998) Allergen immunotherapy: therapeutic vaccines for allergic diseases. A WHO position paper. J Allergy Clin Immuno 102: 558-562.

7. Kleine-Tebbe JJ, Bufe A, Ebner C, Eigenmann P, Friedrich F, et al. (2009) German S-2-Guideline Specific immunotherapy at IgE-mediated allergic diseases. Allergo J 18: 508-537

8. Reich K, Gessner C, Kroker K, Schwab JA, Pohl W, et al. (2011) Immunologic effects and tolerability profile of in-season initiation of a standardized-quality grass allergy immunotherapy tablet: a phase III, multicenter, randomized, double-blind, placebo-controlled trial in adults with grass pollen-induced rhinoconjunctivitis. Clin Ther 33: 828-840.

9. Klimek L, Wolf H, Pfaar O, Wüstenberg E (2010) Intra-seasonal short-time updosing with SQ-standardised subcutaneous immunotherapy in patients with intermittent allergic rhinitis: a new therapeutic option. Allergy 65: 264.
10. Alutard SQ (2010) Summary of product characteristics. Wedel, Germany: ALK

11. Frew AJ, Powell RJ, Corrigan CJ, Durham SR; UK Immunotherapy Study Group (2006) Efficacy and safety of specific immunotherapy with SQ allergen extract in treatment-resistant seasonal allergic rhinoconjunctivitis. J Allergy Clin Immunol 117: 319-325.

12. Zenner HP, Baumgarten C, Rasp G, Fuchs T, Kunkel G, et al. (1997) Short-term immunotherapy: a prospective, randomized, double-blind, placebo-controlled multicenter study of 16 molecular standardized grass and rye allergens in patients with grass pollen-induced allergic rhinitis. J Allergy Clin Immunol 100: 23-29.

13. Hauswald B, Wolf H, Becker F, Becker S, Schnitker J, et al. (2013) Tolerability of a new fast up-dosed immunologically enhanced subcutaneous immunotherapy formulation with optimised allergen/adjuvant ratio under routine practice conditions-A non-interventional observational study. J Invest Allergol Clin Immunol. [Accepted for publication] 